\title{
Oclusão arterial aguda de stent fêmoro-poplíteo
}

\author{
Acute femoropopliteal artery stent obstruction \\ Fabio Henrique Rossi ${ }^{1}$, Milton Kiyonory Uehara ${ }^{2}$, Juliana Chen ${ }^{2}$, Thiago Emilio Burza Maia ${ }^{2}$, \\ Eduardo Mulinari Darold ${ }^{2}$, Andréia Silveira Martins ${ }^{2}$, Nilo Mitsuro Izukawa ${ }^{3}$, \\ Akash Kuzhiparambil Prakasan ${ }^{2}$
}

\section{Resumo}

A oclusão aguda de stent fêmoro-poplíteo pode ser causa de isquemia crítica dos membros inferiores. A terapia fibrinolítica pode não ser a forma de tratamento mais indicada para o grupo de pacientes com esse quadro clínico. Neste artigo, apresentamos um caso em que a retirada de um fragmento de stent por endarterectomia tornou possível a revascularização do membro.

Palavras-chave: Isquemia, extremidade inferior, fibrinólise, endarterectomia, oclusão de enxerto vascular.

\section{Introdução}

A oclusão arterial aguda dos membros inferiores pode ser um importante fator de morbimortalidade cardiovascular. Ocorrem 17 novos casos por 100.000 habitantes/ano ${ }^{1}$. Sua etiologia está relacionada à embolização ou à trombose arterial. Com o aumento da utilização de técnicas endovasculares no tratamento desse grupo de pacientes, devemos considerar a hiperplasia intimal, que pode ser responsável pela trombose do stent no diagnóstico diferencial. A utilização de trombolíticos pode não ser a melhor opção terapêutica nesses casos.

\section{Relato de caso}

Paciente de 70 anos, sexo masculino. Apresentava história de angioplastia com colocação de stent em artéria poplítea esquerda realizada há aproximadamente 1 ano. Foi atendido no Pronto-Socorro, apresentando quadro de claudicação limitante do membro inferior esquerdo desde o dia anterior. Antecedentes pessoais: hipertensão arterial - que se apresentava controlada no momento da internação - e

\begin{abstract}
Femoropopliteal stent obstruction may be responsible for acute lower limb ischemia. Fibrinolytic treatment may not be the best therapeutic approach in this group of patients. We report a clinical case in which removal of a stent fragment with endarterectomy enabled limb revascularization.
\end{abstract}

Key-words: Ischemia, fibrinolysis, endarterectomy, graft occlusion.

tabagismo. Ao exame físico, apresentava apenas o pulso femoral palpável em membro inferior esquerdo e todos os pulsos palpáveis no membro contralateral. Havia ainda: intensa frialdade a partir do terço médio da perna esquerda; diminuição da perfusão plantar; parestesia leve de antepé; e ausência de fluxo arterial em artérias tibiais ao Doppler portátil. A oclusão arterial aguda foi classificada como de Grau 2a de Rutherford. Inicialmente, o paciente foi submetido a aquecimento do membro, sendo realizada heparinização plena endovenosa. Na arteriografia por subtração digital, evidenciaram-se artérias femoral comum, superficial e profunda pérvias com ateromatose difusa não obstrutiva ao fluxo e oclusão arterial no nível do stent localizado em artéria poplítea, com reenchimento de artéria poplítea infragenicular, artérias tibiais e fibular, que apresentavam ateromatose difusa (Figura 1).

Optou-se por trombólise química através da infusão locorregional de estreptoquinase com o auxílio de cateter multiperfurado, posicionado através da técnica de cross-

\footnotetext{
1. Cirurgião vascular assistente, Instituto Dante Pazzanese de Cardiologia (IDPC), São Paulo, SP. Cirurgião vascular e endovascular responsável, Hospital Adventista de São Paulo, São Paulo, SP, e Hospital São Caetano, São Caetano do Sul, SP. Doutor em Ciências (Clínica Cirúrgica), Faculdade de Medicina, Universidade de São Paulo, São Paulo, SP. Especialista em Cirurgia Vascular, SBACV.

2. Setor de Cirurgia Vascular Periférica, IDPC, São Paulo, SP.

3. Chefe, IDPC, São Paulo, SP.

Não foram declarados conflitos de interesse associados à publicação deste artigo.

Artigo submetido em 08.09.08, aceito em 23.03.09.

J Vasc Bras. 2009;8(3):267-270.

Copyright (C 2009 by Sociedade Brasileira de Angiologia e de Cirurgia Vascular
} 


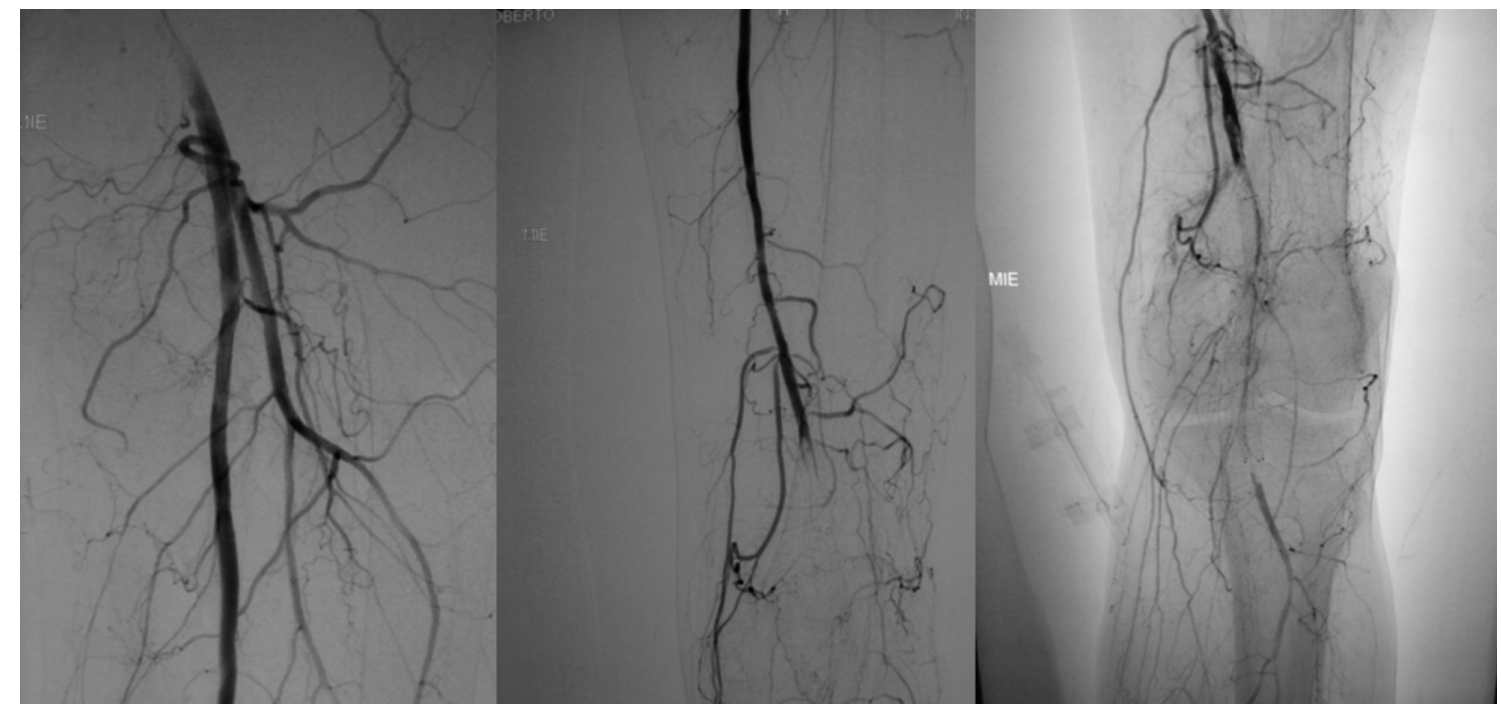

Figura 1 - Arteriografia com subtração digital pré-operatória

over (punção femoral retrógrada contralateral e uso de bainha longa curva). A dose de ataque foi de 20.000 UI em 20 minutos; posteriormente, realizou-se infusão contínua com bomba de infusão na velocidade de $5.000 \mathrm{UI} / \mathrm{h}$. A heparinização sistêmica foi mantida através de infusão endovenosa de heparina não fracionada com bomba de infusão. Os níveis de anticoagulação foram monitorados através dos valores do tempo de tromboplastina parcial ativada (TTPa). Realizou-se arteriografia de controle a cada 6 horas. Após 11 horas de infusão, evidenciou-se queda de hemoglobina, hematócrito e fibrinogênio, não acompanhada de repercussão hemodinâmica. Na arteriografia, realizada através da bainha introdutora, o stent permanecia ocluído (Figura 2).

Não houve alterações clínicas perceptíveis no membro isquêmico durante o período de infusão do fibrinolítico. O paciente foi submetido a exploração cirúrgica. Verificou-se, na ocasião da arteriotomia da artéria poplítea infragenicular, que as malhas do stent tinham avançado até o local escolhido para a realização da anastomose distal do enxerto. No interior do stent, constatou-se a presença macroscópica de oclusão da luz do mesmo por tecido fibroso e esbranquiçado, sugerindo oclusão por processo de hiperplasia intimal (posteriormente confirmado por exame histológico). Após seção da extremidade distal do stent e extração conjunta do material fibroso contido em seu interior por técnica de endarterectomia aberta, foi possível a confecção da anastomose distal e o término do enxerto fê-

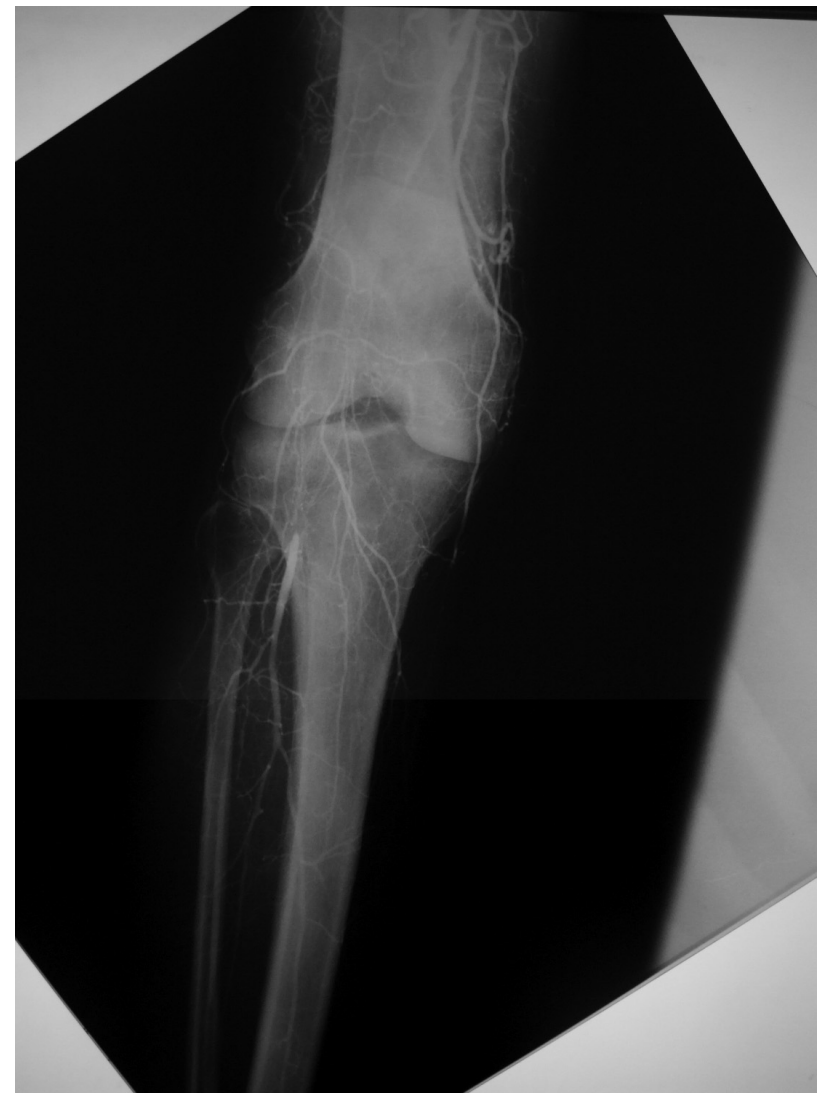

Figura 2 - Arteriografia convencional após 11 horas de infusão do fibrinolítico

moro-poplíteo infragenicular com veia safena magna reversa (Figura 3).

No pós-operatório imediato, o paciente evoluiu, apresentando melhora da perfusão do membro e presença de 


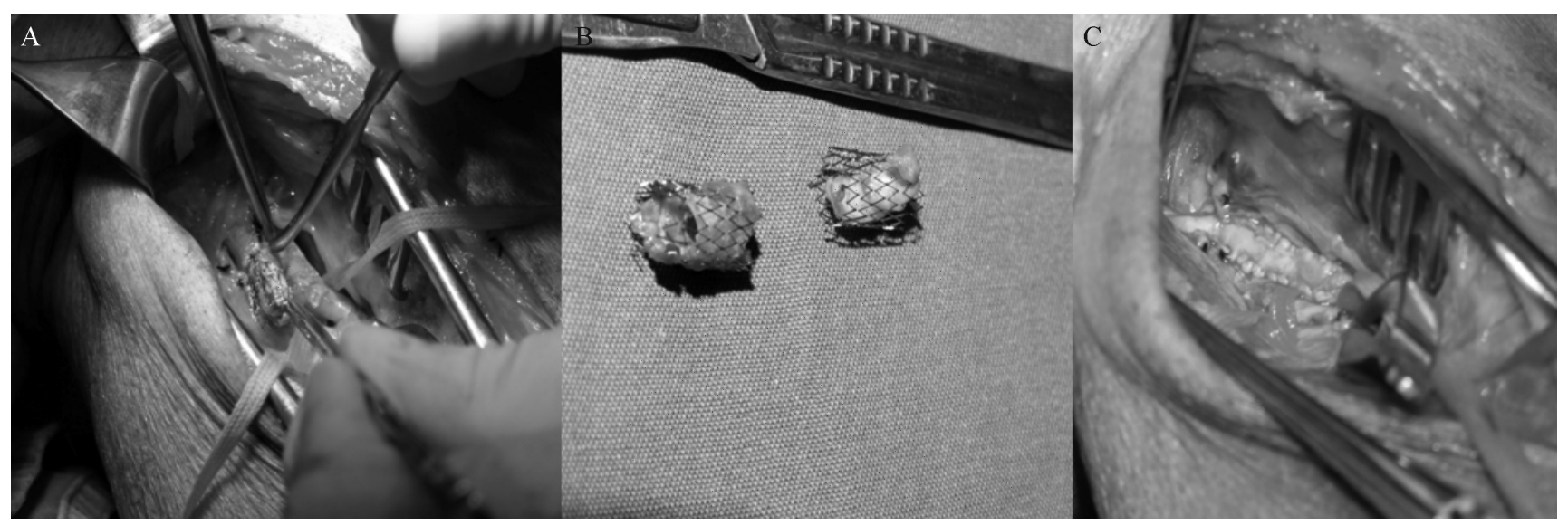

Figura 3 - A) Endarterectomia de fragmento de stent de artéria poplítea; B) fragmentos do stent com material fibrótico em seu interior; C) anastomose distal

pulsos distais, e obteve alta no terceiro dia de pós-operatório, sem intercorrências e melhora dos sintomas.

\section{Discussão}

No presente caso, a oclusão do stent ocorreu aproximadamente 1 ano após o implante, período em que a hiperplasia intimal é a principal causa de oclusão $0^{1,2}$. Estudos experimentais demonstraram que ela ocorre devido a distúrbios hemodinâmicos que existem no local de sua formação - forças de cisalhamento, diferenças de complacência, interação entre componentes do sangue (plaquetas, linfócitos) e o endotélio. A injúria endotelial é o fator final e principal em seu desenvolvimento. Envolve a formação de tecido composto por células musculares lisas e de uma matriz extracelular constituída de tecido conectivo frouxo ${ }^{3}$.

$\mathrm{Na}$ angioplastia fêmoro-poplítea seguida de implante do stent, as características anatômicas e mecânicas locais parecem predispor esse território em especial à hiperplasia intimal ${ }^{4}$, sendo este o principal fator responsável pelos baixos índices de perviedade obtidos 5,6 .

A necessidade da identificação e tratamento precoce de lesões obstrutivas que possam colocar em risco a perviedade da revascularização, seja ela cirúrgica convencio$\mathrm{nal}^{7}$ ou endovascular ${ }^{8}$, já foi bem demonstrada. A correção dessas lesões prolonga a perviedade da revascularização e diminui a morbimortalidade.

$\mathrm{Na}$ ocorrência de trombose aguda, a utilização de fibrinolíticos pode levar à dissolução do trombo recente e revelar a lesão obstrutiva responsável. Isso pode tornar possível o tratamento endovascular da lesão ou diminuir a extensão da cirurgia necessária. Essa afirmação se faz ver- dadeira quando observamos os resultados obtidos no território aortoilíaco ${ }^{9}$. Não encontramos, porém, estudos que justifiquem essa abordagem no território fêmoro-poplíteo.

Não encontramos também referência à secção de fragmento do stent e endarterectomia para possibilitar a realização da anastomose nesse segmento, conforme utilizada em nosso paciente. A extremidade distal do stent localizava-se na artéria poplítea infragenicular. Caso não fosse possível a secção cirúrgica desse fragmento, a anastomose teria que ser realizada em uma das artérias da perna, aumentando muito a morbidade cirúrgica e, possivelmente, diminuindo sua perviedade.

Na ocorrência de oclusão arterial aguda em pacientes submetidos a angioplastia e colocação de stent no território fêmoro-poplíteo, observamos que a fibrinólise pode não ser a melhor opção e não deve atrasar a realização do restabelecimento cirúrgico da perfusão arterial do membro inferior isquêmico. Sabemos que, após o período inicial do implante do stent, falhas técnicas, resistência do leito distal e hipercoagulabilidade podem ser responsáveis pela falência precoce da angioplastia. Além disso, sabemos que o principal mecanismo responsável pela oclusão no primeiro ano de seguimento clínico é a hiperplasia intimal. É nesse período que ocorreram os sintomas isquêmicos de nosso paciente, quando a trombólise pode não ser a melhor opção terapêutica. Além disso, devemos considerar que, diferentemente do território aortoilíaco, as características mecânicas e de extensão da obstrução observadas no território fêmoro-poplíteo desfavorecem a utilização de técnicas endovasculares. 
Concluímos, dessa forma, que a endarterectomia cirúrgica com retirada de fragmento da malha do stent demonstrou ser uma opção terapêutica factível e que pode ser utilizada em pacientes portadores de stent fêmoro-poplíteo que evoluem com oclusão aguda, ou seja, quando a presença do stent pode dificultar a confecção das anastomoses do enxerto.

\section{Referências}

1. Shamoun F, Sural N, Abela G. Peripheral artery disease: therapeutic advances. Expert Rev Cardiovasc Ther. 2008;6:539-53.

2. Kent KC, Liu B. Intimal hyperplasia - still here after all these years! Ann Vasc Surg. 2004;18:135-7.

3. Chervu A, Moore WS. An overview of intimal hyperplasia. Surg Gynecol Obstet. 1990;171:433-47.

4. Sullivan TM, Ainsworth SD, Langan EM, et al. Effect of endovascular stent strut geometry on vascular injury, myo- intimal hyperplasia, and restenosis. J Vasc Surg. 2002;36:143-9.

5. Bosiers M, Deloose K, Verbist J, Peeters P. Present and future of endovascular SFA treatment: stents, stent-grafts, drug coated balloons and drug coated stents. J Cardiovasc Surg (Torino). 2008;49:159-65.

6. Schmehl J, Tepe G. Current status of bare and drug-eluting stents in infrainguinal peripheral vascular disease. Expert Rev Cardiovasc Ther. 2008;6:531-8.

7. Muller-Hulsbeck S, Order BM, Jahnke T. Interventions in infrainguinal bypass grafts. Cardiovasc Intervent Radiol. 2006;29:17-28.

8. Mewissen MW. Stenting in the femoropopliteal arterial segment. Tech Vasc Interv Radiol. 2005;8:146-9.

9. Mussa FF, Peden EK, Zhou W, Lin PH, Lumsden AB, Bush RL. Iliac vein stenting for chronic venous insufficiency. Tex Heart Inst J. 2007;34:60-6.

Correspondência:

Fabio Henrique Rossi

Rua Joaquim Nabuco, 316, sala 94, Bairro Santo Antônio

CEP 09530-120 - São Caetano do Sul, SP

E-mail: vascular369@hotmail.com 